Check for updates

NHS Providers

Cite this as: $B M J 2022 ; 376: 072$ http://dx.doi.org/10.1136/bmj.o72 Published: 12 January 2022

\title{
Sustaining high quality care in a time of transition: is risk management getting enough attention?
}

\section{Catherine Harrison programme development manager}

In any large scale change, there is always increased risk to manage. Risk cuts across all domains including finance and performance, but in healthcare, it is arguably quality of care where it matters most. In the context of the strategic change affecting the health and care system as Integrated Care Systems (ICSs) are established, is risk getting the attention it deserves? How might quality oversight be directly affected systemically and organisationally, as system working becomes more embedded?

As ICSs prepare for statutory status in July 2022, delayed from April, the national bodies have been focused on developing the accompanying policy frameworks in support of this fundamental shift. It's important that this guidance considers and reacts to the current context and the realities of delivering care today.

There has been a growing understanding over these past years that in such deeply complex and dynamic conditions as those faced in healthcare, there are times when no one knows the answers. It's so often been the relationships between trust leaders, their staff and patients that has helped map a way through. Adaption and innovation for the benefit of patients has relied on the ability to collaborate and openly communicate on risk identification, management, and problem-solving.

The National Quality Board's Guidance for ICSs helpfully mentions risk, but the broader narrative and majority of guidance has generally focused on the opportunities that lie in the four expected outcomes of ICSs. It has taken a lot to get Sustainability and Transformation Plans and then ICSs off the ground after years of discussion about integration, but risk management is core to good governance so locally and nationally, we shouldn't shy away from constructive challenge in the name of achieving consensus-now is the time for constructive conversations to help navigate through expected and unexpected challenges.

Worthy of more attention are issues including:

- The need for robust risk frameworks that meet the needs of all healthcare sectors. At the moment, it is implied that ICSs need to work through the real complexities that exist around governance and risk. But risk is distributed in different, evolving ways across the system, and so equal consideration by NHS England and NHS Improvement needs to be given to how different sectors and contexts are affected by change or circumstances. All sectors should be engaged to help develop robust frameworks that account for different circumstances and needs, while still providing important scope for local ownership and adaptation.

- We have already heard anecdotal accounts from trust leaders that it is useful to come together in a System Quality Group (SQG) —-these provide an important forum to come together around quality issues in a system - to identify risks as well as opportunities. However, even these may quickly reach the boundaries of what's possible to address in the system itself without government support and national funding. For example, current pressures on the urgent and emergency care pathway, and in mental health and community services require a "whole system" response involving local and national partners. What happens with the insights local partners and trust boards hold, to better create the conditions for successful management or reduction of that risk to quality of care? What is the process for escalating quality concerns across system boundaries?

- There are currently no published quality impact assessments relating to the new guidance emerging. It's notable also that we repeatedly hear the same question from trust leaders when we update on policy: where is the patient in this? Likewise, safety considerations are rarely given centre stage in the narrative. For instance, the guidance around decision making contains principles for effective decisions between partners within a system. One It should be a clear principle that decisions do not compromise the safety of services.

The potential for unintended consequences in a system as complex as healthcare is always high and vigilance is needed from all levels. Few would argue there is frequently an obvious, clear, "right" way through many of the challenges we face when balancing aspirations, outcomes, cost and impact. There are simply choices to be made, informed by experience and the information available in the given moment. COVID-19 shows us regularly that we are collectively planning in the face of uncertainty. If we do so without considering in what ways may we be wrong, by how much, and what can be the outcome of that, then there is only greater risk.

During a time of such significant transition and challenge, risk-and its spread across to all aspects of quality of care-should be transparently high on our agenda. This should be supported by a strong, consistent voice on quality in all health and care bodies, nationally, regionally, and locally, heard and voiced clearly through interactions with the frontline and at every level of the system. 


\section{OPINION}

Provenance and peer review: not commissioned, not peer reviewed

$1 \quad$ NHS. Working together at scale: guidance on provider collaboratives. August 2021.

https://www.england.nhs.uk/wp-content/uploads/2021/06/B0754-working-together-at-scaleguidance-on-provider-collaboratives.pdf 